\title{
Spatially and temporally resolved temperature measurements of plasma generated in percussion drilling with a diode-pumped Nd:YAG laser
}

\author{
M. Ii, T. P. Duffey, ${ }^{\text {a) }}$ and J. Mazumder ${ }^{\text {) }}$ \\ Department of Mechanical Engineering and Applied Mechanics, Center for Laser Aided Intelligent \\ Manufacturing, University of Michigan, Ann Arbor, Michigan 48109
}

(Received 11 February 1998; accepted for publication 10 July 1998)

\begin{abstract}
Results of spectroscopic temperature measurements of the laser-induced plasma generated during percussion drilling with a high power diode-pumped, pulsed Nd:YAG laser are presented. SAE 52100 steel was drilled with varying average powers. Helium and oxygen were each used as the shield gas. Emission spectra were collected with a monochrometer and an intensified charge coupled detector connected to the optical multichannel analyzer. The plasma electron temperatures were calculated from the relative intensities of the spectral lines. The spatial and temporal temperature distributions are presented. Both drilling times and spatial distributions indicate energy absorption by the plasma. () 1998 American Institute of Physics. [S0021-8979(98)02220-8]
\end{abstract}

\section{INTRODUCTION}

Since its first industrial use in the 1960s laser drilling has taken on an increasingly important role in materials processing and manufacturing and numerous aspects of the laser drilling process have been studied. ${ }^{1-5}$ However, the current $\mathrm{Nd}$ :YAG lasers are inadequate for many applications, due to limitations in average power and beam quality. The newest generation of lasers utilizing diode pumping in a slab geometry offers the prospect of multi-kilowatt (average power) $\mathrm{Nd}$ :YAG systems with focused beams within 2-3 times the diffraction limit. Precise control of the diode pump source, in conjunction with $Q$ switching or mode locking, offers flexibility of pulse format, as well. Peak power densities approaching $10^{10} \mathrm{~W} / \mathrm{cm}^{2}$ are accessible with such lasers, resulting in significant changes in the nature of the laser-material and laser-plasma interactions. These changes must be characterized and understood if the capabilities of highbrightness solid state lasers are to be exploited optimally.

The laser employed in this study was the TRW risk reduction laser, ${ }^{5}$ a diode-pumped Nd:YAG slab laser with optional frequency doubling and high beam quality (1.3-1.7 $\times \mathrm{DL}$ ). The maximum average power available is $\sim 500 \mathrm{~W}$ (unstable resonator configuration), which occurs at a repetition frequency of $400 \mathrm{~Hz}$ and a duty cycle of $20 \%$. The pilot process studied was percussion drilling of SAE 52100 steel. Percussion drilling was chosen for two reasons: first, for its simplicity and suitability for studying the laser materials interaction in new intensity regimes and second, because the laser's high beam quality and repetition rate make percussion drilling of small holes an application with an impact on many industrial applications (e.g. drilling cooling channels in turbine blades ${ }^{6}$ and perforation of wing leading surfaces for reduced $\left.\mathrm{drag}^{7}\right)$.

The primary goal of this study is to obtain diagnostic data to enhance understanding of the influence of the laser-

\footnotetext{
a) Present address: Cymer, Inc, San Diego, CA.

${ }^{b)}$ Author to whom correspondence should be addressed.
}

plasma interaction on the percussion drilling process at different intensities and varying shield gases. Processing data for the drilling time will be correlated with emission spectroscopic temperature measurements taken from the laser generated plasma plume.

\section{EXPERIMENT}

The process studied was percussion drilling of SAE 52100 steel using the TRW risk reduction laser. ${ }^{5}$ Previous work ${ }^{8}$ demonstrated that the optimal pulse format for drilling occurs when the laser runs in a "gain-modulated" mode, which produces a burst of pulses in a comb-like pattern with the duration of each burst equal to the length of the laserdiode pump pulse. The modulated pulse format used in this experiment consisted of three "spikes" per burst; each spike was $75 \mathrm{~ns}$ full width half maximum (FWHM) and individual spikes were separated by $20 \mu \mathrm{s}$. The burst repetition rate was limited to $3 \mathrm{~Hz}$ by the data acquisition rate of the diagnostic apparatus. The drilling was performed using a hyperGaussian beam with a spot size at the workpiece of $100 \mu \mathrm{m}$. A cover gas was supplied at a pressure of 60 psi via a concentric nozzle with an exit diameter of $1 \mathrm{~mm}$ and positioned $3 \mathrm{~mm}$ from the surface of the workpiece. Helium and oxygen were the two gases used.

Drilling experiments were performed with laser output at the fundamental $(1.064 \mu \mathrm{m}) \mathrm{Nd}: \mathrm{YAG}$ laser wavelength. The laser fluence was varied through the use of a rotatable halfwave plate located between a pair of thin film polarizers. The average power was varied from 25 to $200 \mathrm{~mW}$, resulting in peak power densities ranging from on the order of $10^{6}$ to $10^{7} \mathrm{~W} / \mathrm{cm}^{2}$. A quarter wave plate imposed circular polarization on the final processing beam.

The thickness of the SAE 52100 workpiece was 0.76 $\mathrm{mm}$. The total drilling time was measured by the number of pulses required for full penetration. Full penetration was detected using an infrared (IR) fluorescent card placed behind the workpiece. 


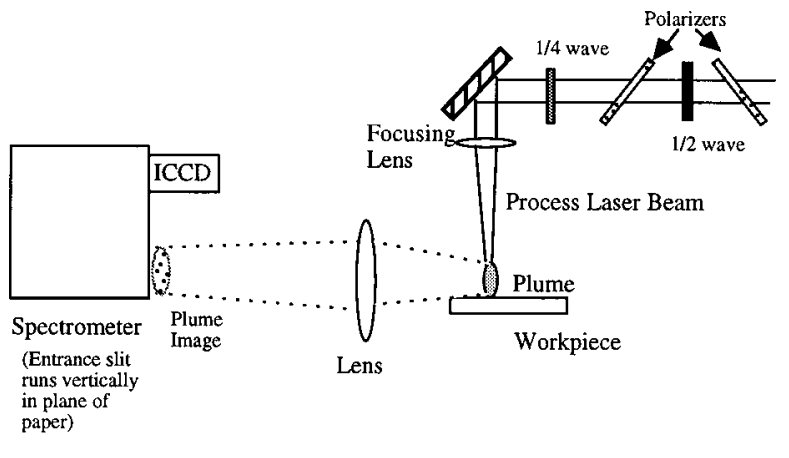

FIG. 1. Layout for diagnostic experiments.

Emission spectra were taken from the plasma plume generated during the drilling process using a Princeton Instruments intensified charge coupled device (ICCD) camera mounted in the image plane of a ISA HR320 spectrometer fitted with a 2400 groove $/ \mathrm{mm}$ grating. The entrance slit width was set to $25 \mu \mathrm{m}$ for maximum resolution (matching the CCD spacing on the ICCD). The detector array was binned into groups of ten along the vertical direction. Light was imaged onto the spectrometer entrance slit by a single $150 \mathrm{~mm}$ focal length fused silica lens of $50 \mathrm{~mm}$ diameter. The lens positioned yielded a magnification factor of 3 between the image and object. The pixel binning, the imaging system magnification, and the internal (vertical) magnification of the spectrometer combined to give imaging spatial resolution of $137 \mu \mathrm{m}$ and a total coverage of $5.2 \mathrm{~mm}$. The spectrometer was oriented with the plume axis parallel to the entrance slit and the image positioned with its axis on the entrance slit. Figure 1 is a schematic of the experimental setup.

The acquisition gate time on the spectrometer was set to 50 ns. Emission spectra were acquired only after the first spike in each burst, due to limitations on the retriggering rate of the gate circuitry. Ten acquisitions were accumulated for each spectrum to improve signal to noise and average out the effects of shot-to-shot variations. The emission spectra were taken over the first ten bursts in the drilling process. The gate was triggered by a photodiode monitoring reflected laser light from the second thin film polarizer. Delay of the gate opening relative to the trigger was varied from 50 to $500 \mathrm{~ns}$. Spectra were taken over a wavelength range of 419-432 nm. Data were taken over eight time delays: 0, 50, 100, 150, 200, 250, 300, and $500 \mathrm{~ns}$ after the peak of the first spike in each pulse. When helium was used as the assist gas, spectra taken with gate delays of less than $100 \mathrm{~ns}$ (i.e., during the strongest part of the spike) exhibited continuum rather than line emission. For oxygen assist gas, delays of less than $150 \mathrm{~ns}$ did not provide discernible emission lines.

A typical spectrum for the 419-432 spectral region as well as the neutral Fe I lines analyzed are shown in Fig. 2.

Seven neutral iron lines were identified for detailed study to obtain temperature distributions. The specifications of these lines from National Bureau of Standards (NBS) data ${ }^{9}$ are displayed in Table I.

Temperature estimates are calculated from the integrated line intensities according to the local thermodynamic equi-

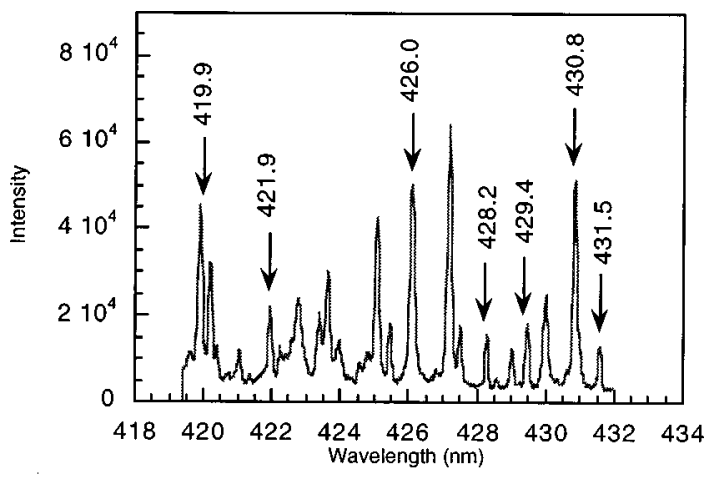

FIG. 2. Typical spectrum for the 419-432 nm region.

librium (LTE) assumption and the Boltzmann relation:

$$
\ln \left|\frac{I_{1} \lambda_{1}^{3} \cdot g_{2} A_{2}}{g_{1} A_{1} \cdot I_{2} \lambda_{2}^{3}}\right|=\frac{E_{2}-E_{1}}{k T},
$$

where $I_{i}, A_{i}, g_{i}, E_{i}$, and $\lambda_{i}$ are, respectively, the integrated line intensity, spontaneous decay rate, level degeneracy, upper level energy, and wavelength of the electronic transition associated with each spectral line, $i$. When the upper level energy divided by the Boltzmann constant is plotted versus $\ln \left(I \lambda^{3} / g A\right)$ and the data for all the Fe I lines is fit to a straight line using linear regression, the resulting slope is used to determine the temperature via the relationship:

$$
T=-\frac{1}{\text { slope }} \text {. }
$$

Figure 3 shows this process of temperature determination when oxygen is used as a cover gas $(50 \mathrm{~mW}$ average power, $200 \mathrm{~ns}$ delay, $0 \mathrm{~mm}$ from the workpiece surface). The standard deviation of the slope is used to estimate the uncertainty in the temperature measurements.

\section{RESULTS AND DISCUSSION}

Figure 4 shows the drilling time (expressed in terms of number of pulses) required to achieve full penetration of a $0.76 \mathrm{~mm}$ thick SAE 52100 workpiece as a function of average power with helium and oxygen as cover gases. The data points represent average drilling times, the error bars $\pm 1 \mathrm{sd}$.

For both cover gases, the number of pulses needed to drill through decreases as the average power is increased but the improvement in drilling time is not proportional to the increase in power. The data suggest the second order poly-

TABLE I. Data on spectral lines.

\begin{tabular}{ccc}
\hline \hline Wavelength $(\mathrm{nm})$ & $\begin{array}{c}\text { Energy of upper } \\
\text { level }\left(\mathrm{cm}^{-1}\right)\end{array}$ & $\begin{array}{c}g A \\
\text { (degeneracy } \times \mathrm{A} \\
\text { coefficient) } 10^{7} / \mathrm{s}\end{array}$ \\
\hline 419.910 & 48383 & 25.0 \\
421.936 & 52514 & 27.0 \\
426.048 & 42816 & 15.0 \\
428.241 & 40895 & 2.0 \\
429.413 & 35257 & 0.71 \\
430.791 & 35768 & 5.9 \\
431.509 & 40895 & 1.5 \\
\hline \hline
\end{tabular}




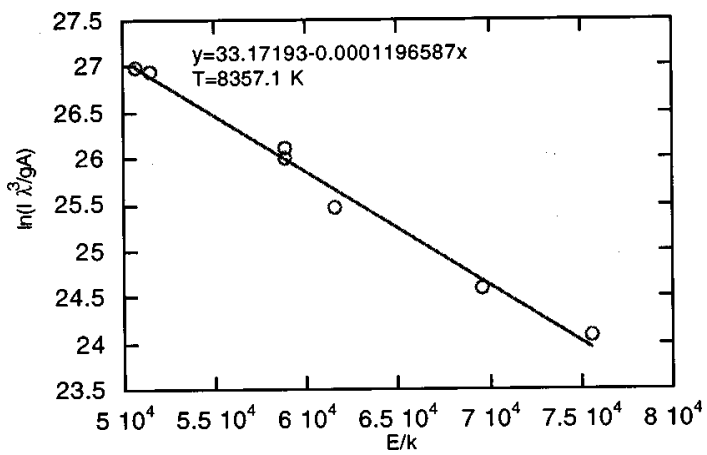

FIG. 3. $\ln \left(I \lambda^{3} / g f\right)$ vs $E / k(50 \mathrm{~mW}$ average power, $200 \mathrm{~ns}$ delay, $0 \mathrm{~mm}$ from the workpiece surface, oxygen cover gas).

nomial curve fit shown in Fig. 4. Although the curve fit agrees with the helium data with a correlation coefficient, $R=0.98$ and with oxygen data with $R=0.90$, no theoretical explanation is offered to justify such a fit. A shorter drilling time with oxygen assist gas is expected and has been reported elsewhere. ${ }^{10}$

Figures 5-11 show the time evolution of the plasma electron temperature for fixed average power levels with helium as the cover gas. Standard deviations for the derived temperature values were approximately $100-500 \mathrm{~K}$. They are omitted from the figures for clarity.

For all power levels temperature decreases as delay increases: the plasma is cooling as would be expected. For all powers, the plasma appears to reach a uniform temperature by $500 \mathrm{~ns}$. A more definite overall temporal characterization of the plasma is hindered by difficulties in interpreting weaker spectral lines.

Figure 12 shows the change in spatially averaged temperature as delay time progresses. There seems to be a nearly constant cooling rate throughout the 100-500 ns delay range for all power levels. Between powers, the cooling rates vary from 3 to $6 \times 10^{9} \mathrm{~K} / \mathrm{s}$ with a mean of $4 \times 10^{9} \mathrm{~K} / \mathrm{s}$. This average cooling rate may hold for all powers, with measured individual variations in the data due to experimental error.

Figure 13 shows the average temperature versus height for different powers. As would be expected, the plasma temperature increases as average laser power increases for all delays. The rise in temperature with increasing power seen in Fig. 13 suggests that the less than proportional decrease in

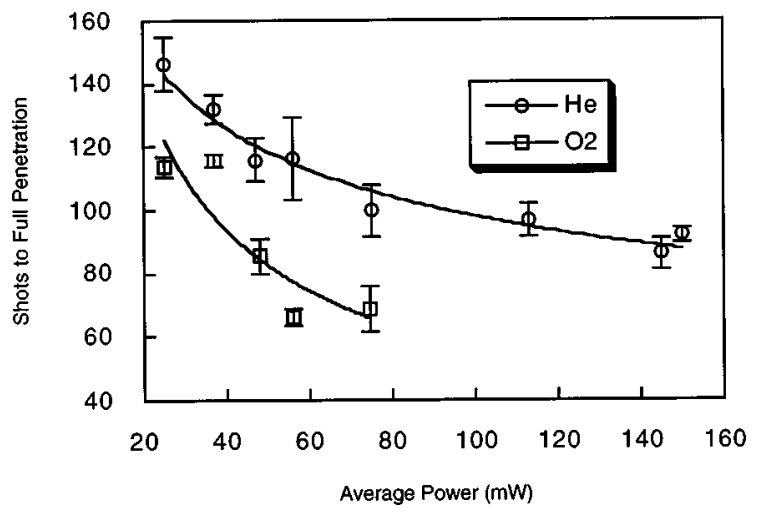

FIG. 4. Drilling time vs average power.

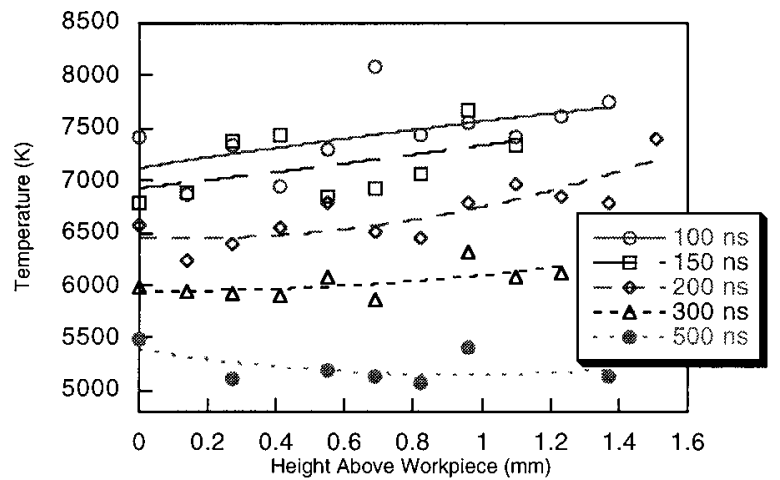

FIG. 5. Temperature vs height above workpiece $(25 \mathrm{~mW}$ ave power, $\mathrm{He}$ cover gas).

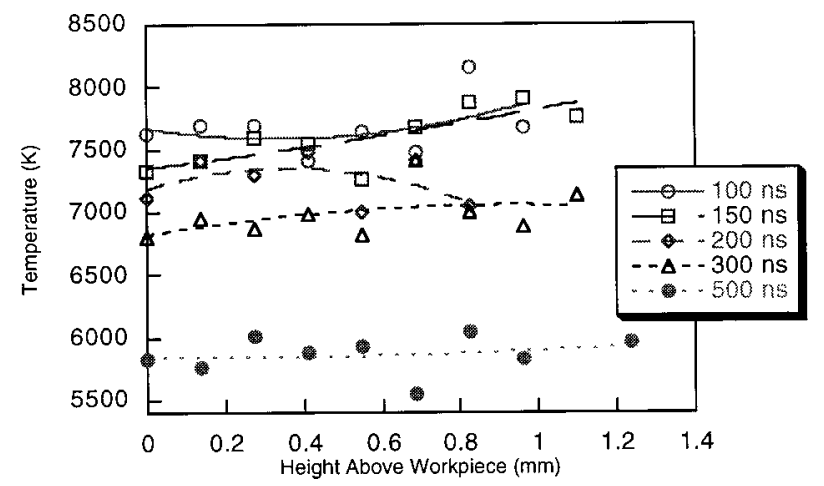

FIG. 6. Temperature vs height above workpiece $(37 \mathrm{~mW}$ ave power, $\mathrm{He}$ cover gas).

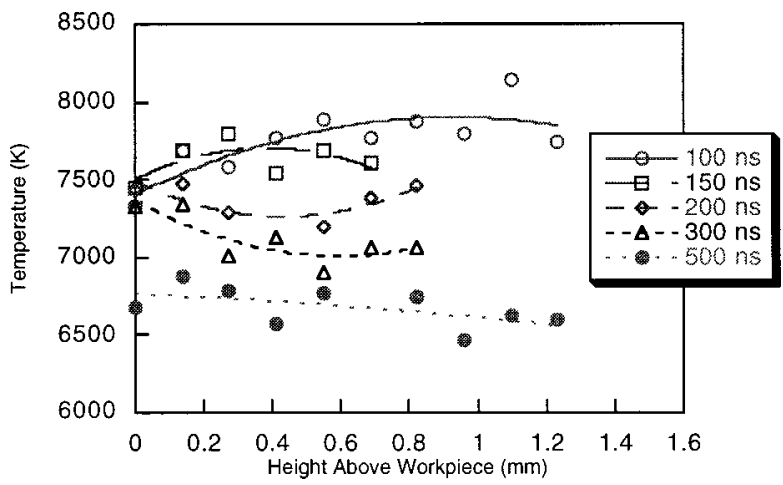

FIG. 7. Temperature vs height above workpiece $(50 \mathrm{~mW}$ ave power, $\mathrm{He}$ cover gas).

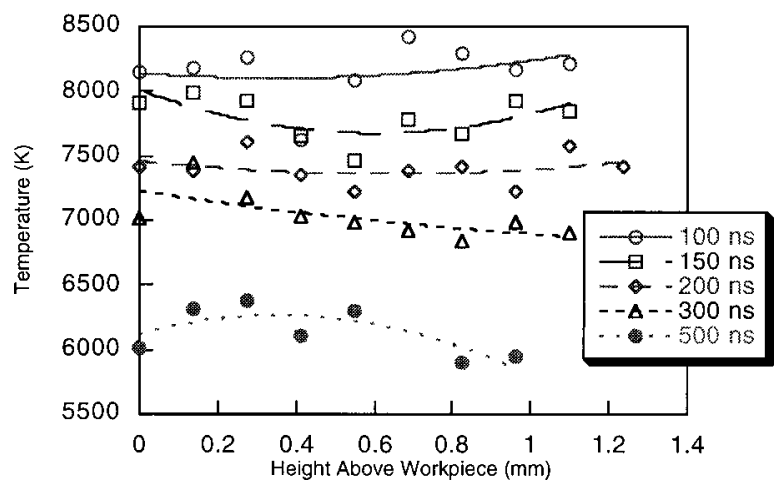

FIG. 8. Temperature vs height above workpiece $(75 \mathrm{~mW}$ ave power, $\mathrm{He}$ cover gas). 


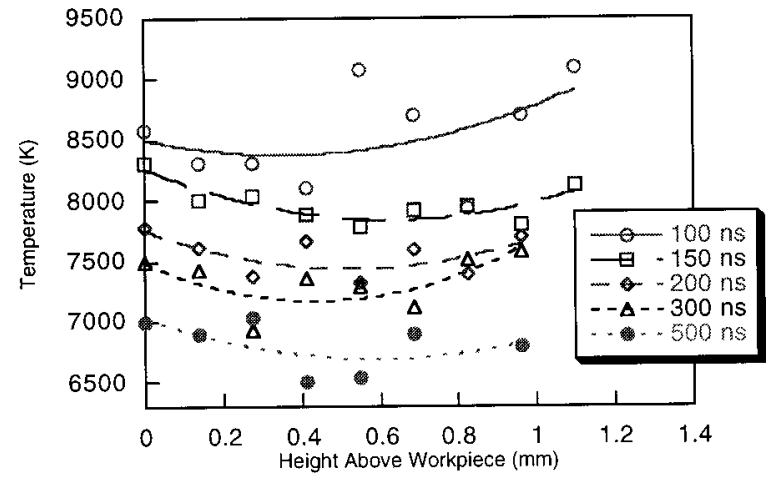

FIG. 9. Temperature vs height above workpiece $(100 \mathrm{~mW}$ ave power, $\mathrm{He}$ cover gas).

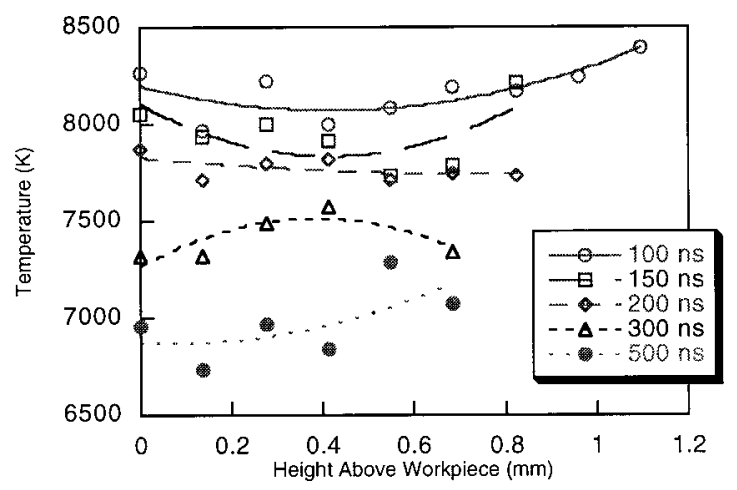

FIG. 10. Temperature vs height above workpiece $(150 \mathrm{~mW}$ ave power, $\mathrm{He}$ cover gas).

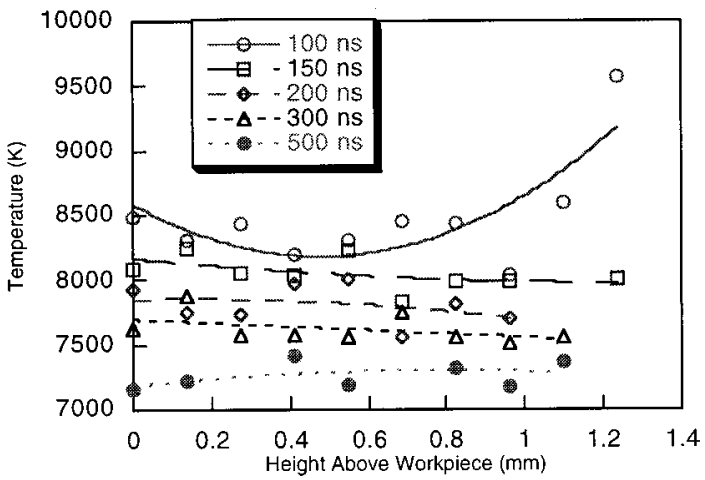

FIG. 11. Temperature vs height above workpiece $(200 \mathrm{~mW}$ ave power, $\mathrm{He}$ cover gas).

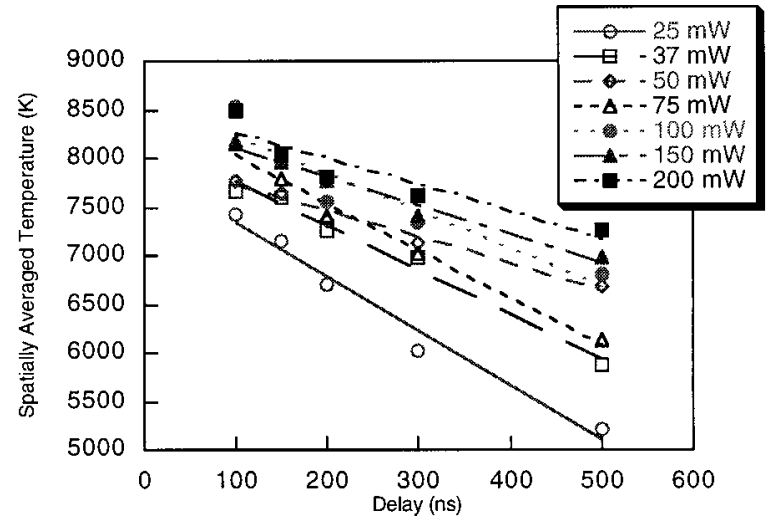

FIG. 12. Spatially averaged temperature vs delay (He cover gas).

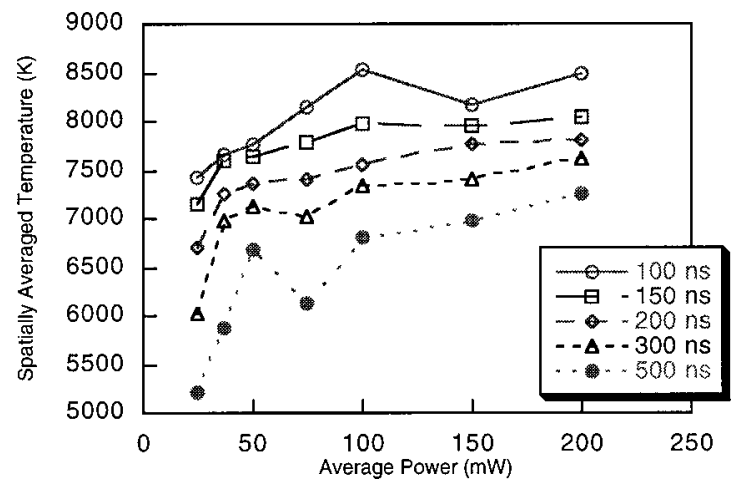

FIG. 13. Spatially averaged temperature vs average power (He cover gas).

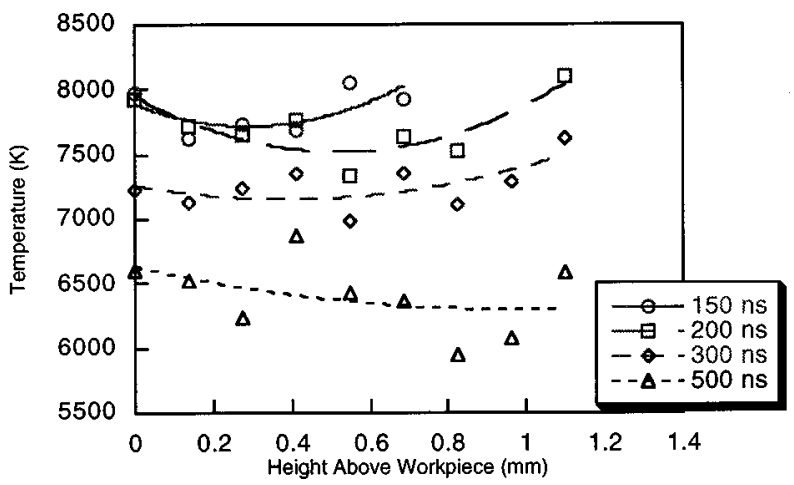

FIG. 14. Temperature vs height above workpiece $\left(25 \mathrm{~mW}\right.$ ave power, $\mathrm{O}_{2}$ cover gas).

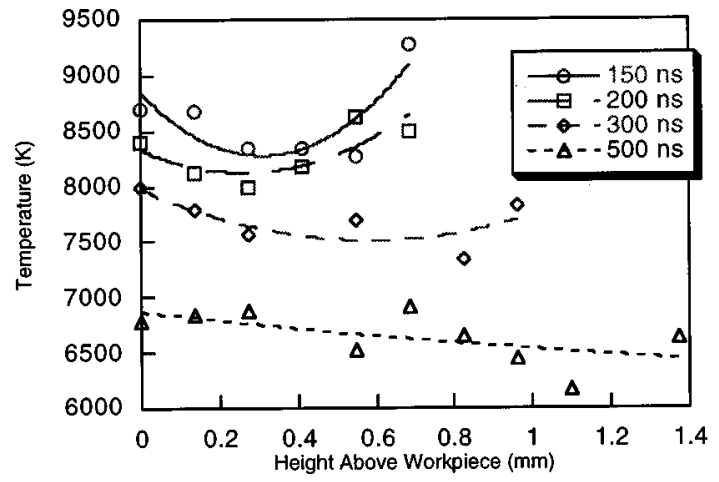

FIG. 15. Temperature vs height above workpiece $\left(37 \mathrm{~mW}\right.$ ave power, $\mathrm{O}_{2}$ cover gas).

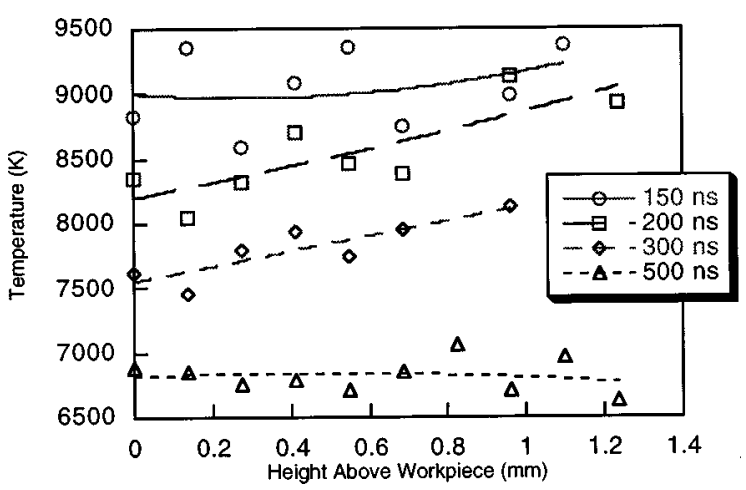

FIG. 16. Temperature vs height above workpiece $\left(50 \mathrm{~mW}\right.$ ave power, $\mathrm{O}_{2}$ cover gas). 


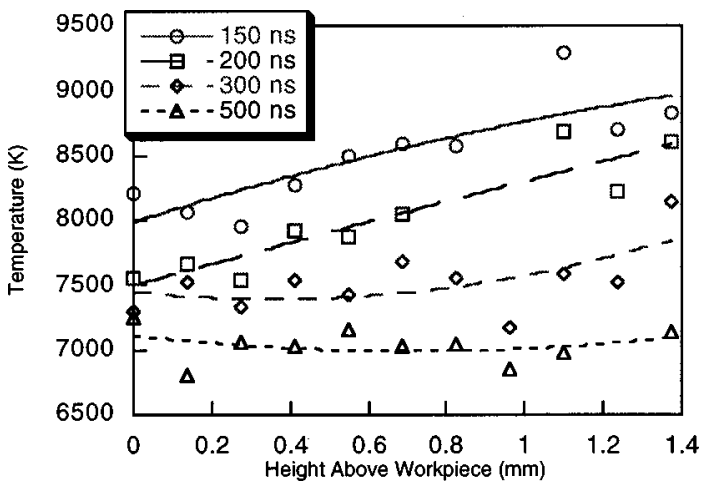

FIG. 17. Temperature vs height above workpiece $\left(75 \mathrm{~mW}\right.$ ave power, $\mathrm{O}_{2}$ cover gas).

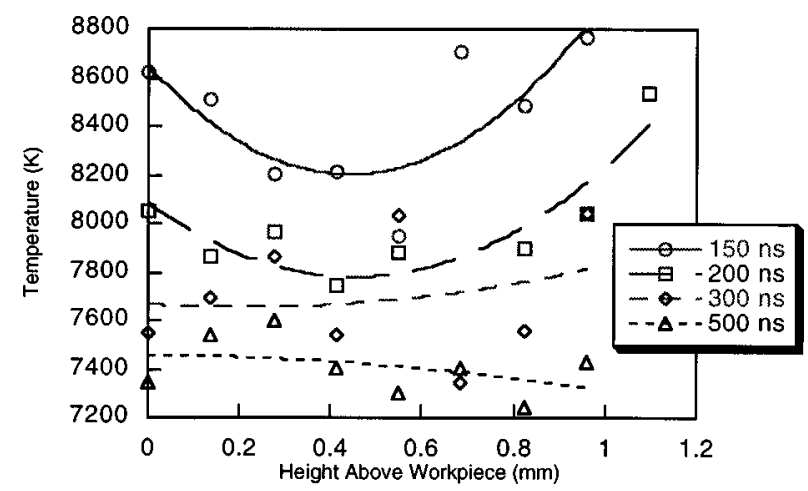

FIG. 18. Temperature vs height above workpiece $\left(100 \mathrm{~mW}\right.$ ave power, $\mathrm{O}_{2}$ cover gas).

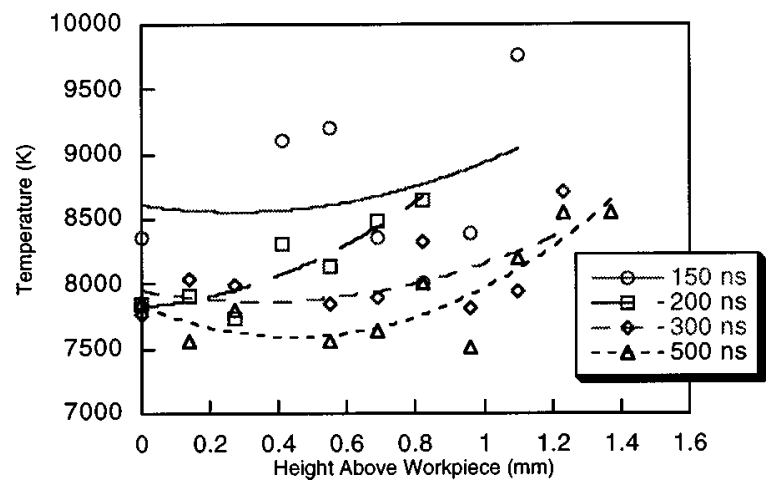

FIG. 19. Temperature vs height above workpiece $\left(150 \mathrm{~mW}\right.$ ave power, $\mathrm{O}_{2}$ cover gas).

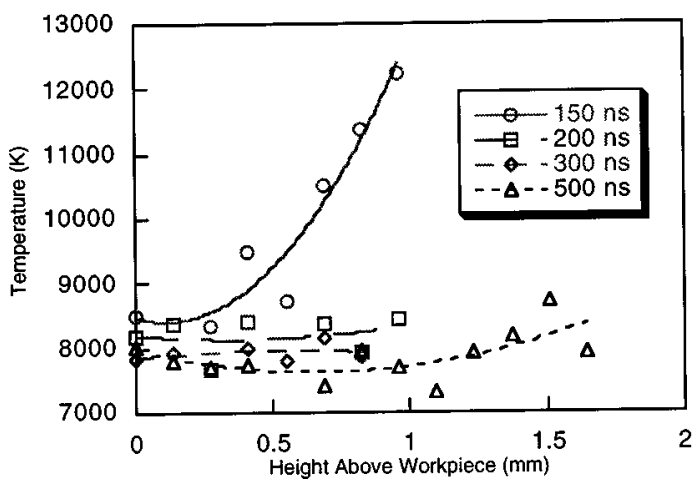

FIG. 20. Temperature vs height above workpiece $\left(200 \mathrm{~mW}\right.$ ave power, $\mathrm{O}_{2}$ cover gas).

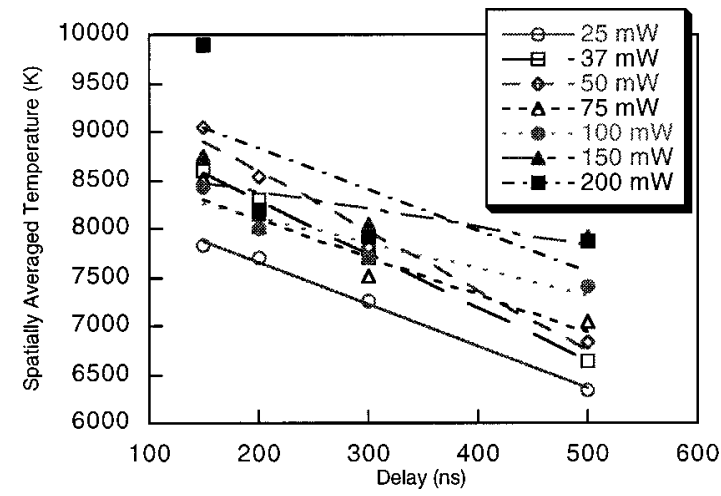

FIG. 21. Spatially averaged temperature vs delay $\left(\mathrm{O}_{2}\right.$ cover gas $)$.

drilling time seen in Fig. 4 may be due in part to energy absorption by the plasma. The rate of increase is much larger-between 25 and $50 \mathrm{~mW}$ - than after. There are also three points where the average temperature decreases after a power increase (after $100 \mathrm{~mW}$ at $100 \mathrm{~ns}$ and after $50 \mathrm{~mW}$ at 300 and $500 \mathrm{~ns}$ ). Anomalies at the $100 \mathrm{~ns}$ delay are probably due to errors in spectral interpretation at the short delay time. These decreases in average temperature after $50 \mathrm{~mW}$ coincide with a decrease in the rate of increase in temperature.

Figures 14-20 show the time evolution of the plasma temperature for fixed average power levels with oxygen as the cover gas. Comparing these figures with those seen for helium, it is seen that the temperature found in the plasma is higher when oxygen is used as the cover gas. When oxygen is the shield gas, the plasma also seems to reach a uniform temperature by $500 \mathrm{~ns}$ for powers less than $150 \mathrm{~mW}$.

Figure 21 shows the time progression of the spatially averaged temperature values. As with helium a constant cooling rate is indicated throughout the $100-500$ ns delay range for all power levels up to $150 \mathrm{~mW}$. The discrepancies seen at the higher power are attributable to the difficulties in interpreting spectra at high powers. Between powers, the cooling rates vary from 2 to $6 \times 10^{9} \mathrm{~K} / \mathrm{s}$ with the same mean value of $4 \times 10^{9} \mathrm{~K} / \mathrm{s}$ as seen with helium. This average cooling rate may hold for both cover gases as well as all powers, with measured individual variations in the data due to experi-

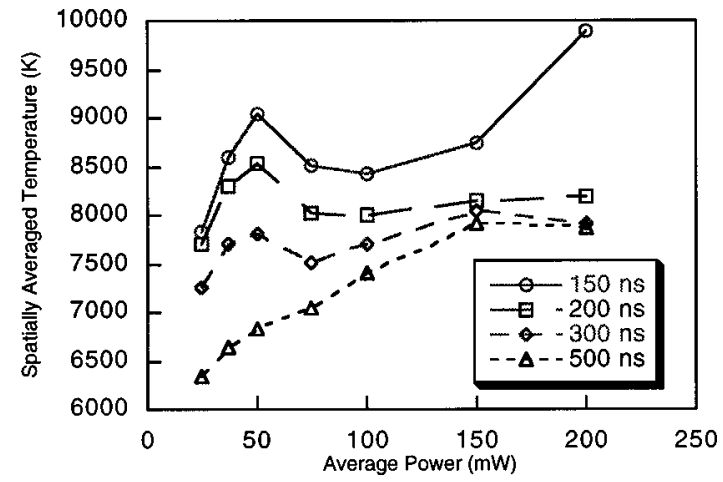

FIG. 22. Spatially averaged temperature vs average power $\left(\mathrm{O}_{2}\right.$ cover gas). 
mental error. Figure 22 shows the average temperature versus height for different powers with oxygen as the shield gas and suggests plasma energy absorption as the helium data did.

\section{CONCLUSIONS}

In this study, we have investigated the process of percussion drilling of SAE 52100 with a diode-pumped $\mathrm{Nd}$ :YAG laser. For a given pulse format, repetition rate, and spot size, the effects of varying average power and shield gas were investigated. It has been found that increasing average laser power decreases drilling time, although the decrease in drilling time is not proportional to the increase in power. Absorption of laser energy by plasma may explain this nonproportional decrease. Oxygen cover gas yielded a lower drilling time than He. The drilling time data fit a second order polynomial curve fit.

The emission spectroscopy indicates that the temperature of the laser induced plasma increases with average power and decreases as delay increases. There seems to be a nearly constant plasma cooling rate of $\approx 4 \times 10^{9} \mathrm{~K} / \mathrm{s}$ for most powers and both cover gases. Plasma temperature is higher with oxygen than with $\mathrm{He}$ as a cover gas.

\section{ACKNOWLEDGMENTS}

This work was conducted under the DARPA Technology Reinvestment Program's Precision Laser Machining Project. The authors also wish to thank Dr. Jason Machan and David Burchman of TRW, Redondo Beach, CA.

${ }^{1}$ J. T. Luxon and D. E. Parker, Industrial Lasers and Their Applications (Prentice-Hall, New York, 1985).

${ }^{2}$ W. W. Duley, Laser Processing and Analysis of Materials (Plenum, New York, 1983)

${ }^{3}$ M. Bass, Laser Material Processing (North-Holland, Amsterdam, 1983).

${ }^{4}$ J. F. Ready, Industrial Applications of Lasers (Academic, New York, 1978).

${ }^{5}$ J. Machan, M. Valley, G. Holleman, M. Mitchell, D. Burchman, J. Zamel, G. Harpole, H. Injeyan, and L. Marabella, J. Laser Appl. 8, 225 (1996).

${ }^{6}$ X. Chen, W. T. Lotshaw, A. L. Ortiz, P. R. Staver, C. E. Erikson, M. H. McLaughlin, and T. J. Rockstroh, J. Laser Appl. 8, 233 (1996).

${ }^{7} \mathrm{~W}$. Maher, a Laser drilling of small holes, in Proceedings of the International Conference on Lasers and Electro-Optics (ICALEO'95), (Laser Institute of America, San Diego, 1995).

${ }^{8}$ T. P. Duffey, P. Mohanty, K. Nagarathnam, M. Ii, and J. Mazumder, Proceedings of the International Conference on Lasers and Electro-Optics (ICALEO'95) (Laser Institute of America, San Diego, 1995), Vol. 80, p. 825.

${ }^{9}$ C. H. Corliss and W. R. Bozman, Experimental Probabilities for Spectral Lines of Seventy Elements, NBS Monograph (U.S. Govt. Printing Office, Washington, DC, 1962), Vol. 53.

${ }^{10}$ R. S. Patel and M. Q. Brewster, J. Heat Transfer 112, 170 (1990). 